Cahiers balkaniques

Balkaniques

36-37| 2008

L'image de la période ottomane dans les littératures balkaniques

\title{
Les Turcs dans les chansons des Rroms des Balkans à l'époque ottomane
}

The Turks in the Rromani songs in the Balkans during the Ottoman period

\section{Marcel Courthiade}

\section{OpenEdition}

\section{Journals}

Édition électronique

URL : https://journals.openedition.org/ceb/1557

DOI : 10.4000/ceb.1557

ISSN : 2261-4184

\section{Éditeur}

INALCO

\section{Édition imprimée}

Date de publication : 1 décembre 2008

Pagination : 243-272

ISBN : 978-2-85831-173-6

ISSN : 0290-7402

\section{Référence électronique}

Marcel Courthiade, «Les Turcs dans les chansons des Rroms des Balkans à l'époque ottomane », Cahiers balkaniques [En ligne], 36-37 | 2008, mis en ligne le 16 mai 2012, consulté le 06 juillet 2021. URL : http://journals.openedition.org/ceb/1557 ; DOI : https://doi.org/10.4000/ceb.1557

Ce document a été généré automatiquement le 6 juillet 2021.

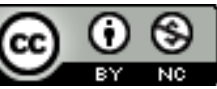

Cahiers balkaniques est mis à disposition selon les termes de la Licence Creative Commons Attribution - Pas d'Utilisation Commerciale 4.0 International. 


\title{
Les Turcs dans les chansons des Rroms des Balkans à l'époque ottomane
}

The Turks in the Rromani songs in the Balkans during the Ottoman period

\author{
Marcel Courthiade
}

1 Dans la mesure où la littérature des Rroms n'a conquis la sphère de l'écrit individuel qu'après la Seconde Guerre mondiale (du moins dans les Balkans, puisqu'en Russie elle a débuté un quart de siècle plus tôt), donc à un moment où l'Empire ottoman avait depuis longtemps disparu, il n'est guère surprenant que l'image des Turcs n'y apparaisse pas. En effet, les confrontations sanglantes des siècles passés n'avaient plus la force de motiver un auteur. D'un autre côté, les relations actuelles entre Rroms et Turcs sont plutôt celles de minorités qui se côtoient sans vraiment interagir sur le plan culturel $^{1}$ et il n'est donc pas non plus surprenant de constater leur absence de la littérature rromani écrite. Rappelons que dans les Balkans cette dernière s'est surtout développée en Macédoine (uniquement tentatives de poésie) et en Cossovie (où l'on trouvait aussi un auteur en prose), sous la forme de petits feuillets souvent manuscrits ou «tapuscrits » circulant dans les «soirées poétiques » fréquentées dans les années 1970-1990 par de très nombreux auteurs en herbe, et en Serbie proprement dite, où les auteurs étaient moins nombreux, mais plus instruits et avaient accès à l'imprimerie. Le dictionnaire des auteurs rroms de (nouvelle) Yougoslavie de Luka Šteković́ ${ }^{2}$ comporte plus de 120 noms d'auteurs - il est vrai qu'il est maximaliste et répertorie tout auteur dont le nom apparaît au moins dans une publication. En revanche les auteurs se comptent sur les doigts d'une main pour l'ensemble des pays voisins : Pëllumb Furtuna (poésie satirique et pour enfants) en Albanie, Sali Ibrahimi (poésie lyrique ou de combat) en Bulgarie et Luminiţa Cioabă (récits et poésie) en Roumanie.

2 C'est donc vers la littérature orale ${ }^{3}$ que l'on doit se tourner pour trouver trace de Turcs. Le corpus qui peut nous apporter des informations à ce point de vue est un recueil d'une centaine de chants rroms de Bulgarie, recueillis dans les années 1950 par Manuś Romanov $^{4}$, scénariste rrom de Sofia - nous verrons qu'une ballade des Rroms de 
Roumanie fait écho à ces chansons. La première série de chants de Bulgarie, intitulée « le temps des beys et des agas" présente un certain nombre de Turcs, dont les traits sont en tout point comparables aux «gendarmes» de la seconde série «le temps des gendarmes ».

\section{Les noms des Turcs}

3 Le terme le plus courant pour désigner les Turcs est Xoraxaj (var. Koxraj) c'est-à-dire musulman - par opposition à Das, le chrétien donc le Bulgare en Bulgarie. Ce terme de Xoraxaj est connu dans pratiquement tous les parlers rroms, même dans le caló ${ }^{5}$ d'Espagne (sous la forme jorajay, graphiée à l'espagnole) où il signifie « Arabe ». Chez les Kabuzi d'Albanie toutefois, c'est l'Albanais que Xoraxaj désigne - de toute manière le musulman. Les féminins respectifs sont Xoraxni, Koxrani et Dasni. On ajoutera comme terme ethnique Aràpi - à la fois nom du «nègre ", souvent esclave du Turc et complice de ses exactions, et nom propre de cheval noir (voir texte $n^{\circ} 5$ ), comme dans la plupart des parlers populaires balkaniques (l'équivalent comme nom de cheval noir est Mùrgo en Roumanie).

Exemples :

«Aj, phrala!len, amala!len,

ćhiven piro, den naśiba,

te uxtavas amaro śero

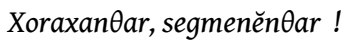

te na ćalavel man dekh Xoraxaj !»

[pièce $\mathrm{n}^{\circ} 1$ ]

«Ah, mes frères, mes amis,

jouez des jambes, prenez la fuite,

pour que nous sauvions notre tête,

des Turcs, des segmènes !6 (Pour ce mot v. infra.)

que quelque Turc ne me frappe pas!

\begin{tabular}{|l|l|}
\hline $\begin{array}{l}\text { So mangen tume amen } \theta \text { ar, be, Xoraxa !len ? } \\
\text { So mangen tume saltě ćorren } \theta \text { ar? ? } \\
\text { [pièce } \mathrm{n}^{\circ} \text { 7] }\end{array}$ & $\begin{array}{l}\text { "Que voulez-vous de nous, les Turcs? } \\
\text { Que voulez-vous de tels miséreux? }\end{array}$ \\
\hline $\begin{array}{l}\text { Ma astar man, Mamud, } \\
\text { Xoraxaja dikhen, } \\
\text { tut ka maren Mamud, } \\
\text { pale man ka dolen.» } \\
\text { [pièce } \mathrm{n}^{\circ} \text { 9] }\end{array}$ & $\begin{array}{l}\text { "Ne me touche pas, Mamoud, } \\
\text { des Turcs regardent, } \\
\text { ils vont te battre Mamoud, } \\
\text { et ensuite se saisiront de moi. » }\end{array}$ \\
\hline
\end{tabular}

L'adjectif est très régulièrement xoraxano (var. koxrano) :

\begin{tabular}{|l|l|}
$\begin{array}{l}\text { Xoraxani cikni chajorri, } \\
\text { dasikani bari bororri! } \\
\text { [pièce } \mathrm{n}^{\circ} 88\end{array}$ & $\begin{array}{l}\text { Petite fillette turque, } \\
\text { grande bru chrétienne! }\end{array}$ \\
\hline
\end{tabular}


Deux autres termes plus obscurs sont ćitàki (bulg. ЧИТАК « Turc (péjoratif) »< tc. cltak, çitik «étranger, nouveau venu, Turc d'Europe, baragouineur " ${ }^{7}$ ) et Manàvi (bulg. МАНАФ[Ин] « turc d'Asie mineure » <tc. manav) :

\begin{tabular}{|l|l|}
\hline $\begin{array}{l}\text { Te si dekh rromorro, } \\
\text { vudar phuter lesqe, } \\
\text { te si dekh ćitàki, } \\
\text { o vudara phande, } \\
\text { lele, mi ćhajorri! } \\
\text { Citàki si beng, } \\
\text { Rakì ka mangel. } \\
\text { [pièce } \mathrm{n}^{\circ} \text { 8] }\end{array}$ & $\begin{array}{l}\text { Sivre-lui la porte, } \\
\text { si c'est quelque Turc, } \\
\text { ferme la porte, } \\
\text { malheur à nous, ma fille! } \\
\text { Le Turc est un démon, } \\
\text { il va exiger de l'eau-de-vie. }\end{array}$ \\
\hline $\begin{array}{l}\text { So bendindăn, Alemo, } \\
\text { katar-o kalo Manàvi? } \\
\text { [pièce } \mathrm{n}^{\circ} \text { 85] }\end{array}$ & $\begin{array}{l}\text { Qu'est-ce qui te plaît, Alema, } \\
\text { chez cet Anatolien moricaud? }\end{array}$ \\
\hline
\end{tabular}

Dans l'un des chants [pièce $n^{\circ} 6$ ] ils sont appelés gazés, terme englobant tous ceux qui ne sont pas Rroms, mais le récit, dans lequel ils sont appelés sultans, montre bien qu'il s'agit de Turcs. Ainsi, ces textes donnent déjà une première image du Turc, seigneur impitoyable, voire cruel, sinon sadique et jouant arbitrairement de la vie et des souffrances de ses victimes. Ils sont en effet toujours représentés comme des potentats plus ou moins locaux: aga, bey, sultans ou segmènes (militaires turcs en service de police, cf. bulg. СЕЙМЕНИН, turc seğmen) et surtout abusant cyniquement de leur prépotence. Parfois ils circulent en potèra (groupe de gens armés, lancés à la chasse de quelqu'un, cf. bulg. ПотЕРЯ). Nulle part il n'est question d'un simple Turc du peuple, nulle part d'un ami ou d'un allié. C'est avant tout la cruauté arbitraire et sans pitié des Turcs (ou de leurs serviteurs nègres, comme l'aràpi de la pièce $n^{\circ}{ }^{5}$ ) qui fait l'objet de nombreux chants (pièces $n^{\circ} 1$ à 9 ).

7 Cette violence, sanglante et sans merci, n'est toutefois pas limitée aux seuls Turcs; en effet, les gendarmes bulgares ne laissent rien à envier aux Ottomans puisque, allant souvent par groupes de douze, ils infligent aux Rroms (mais peut-être au peuple tout entier ?) les traitements les plus injustes - ou du moins les moins compris par leur victime :

\begin{tabular}{|l|l|}
\hline Iklilo o Elmaz & Elmaz est sorti \\
anglal-i pòrtica, & devant le portail, \\
so te dikhel, baba, & que voit-il, père, \\
deśuduj 3andàra, & douze gendarmes, \\
sa nange ćhurença, & tous sabre au clair, \\
ćalade les, baba, & ils l'ont frappé père, \\
deśudo thanen $\theta e$. & en douze endroits. \\
\hline
\end{tabular}

Les Rroms eux-mêmes ne sont pas en reste quant aux sévices sur leurs proches : 


\begin{tabular}{|c|c|}
\hline $\begin{array}{l}\text { Uśtilo o Kòne } \\
\text { maśkare răt, Devl!a, } \\
\text { thaj lia e ćhuri } \\
\text { ćhingărda e Mira } \\
\text { vov ină thanen } \theta e, \\
\text { ină xëvă kerda! }\end{array}$ & $\begin{array}{l}\text { Kone s'est levé } \\
\text { en pleine nuit, Seigneur, } \\
\text { et il a pris un couteau } \\
\text { il a poignardé Mira } \\
\text { et en neuf endroits } \\
\text { il lui a fait neuf trous! }\end{array}$ \\
\hline $\begin{array}{l}\text { Ta ikaldăs mo Śabànos } \\
\text { parni ćhuri dandorrença, bre! } \\
\text { Jekhar dinǎs mo Śabànos, } \\
\text { ikalǎs me Najlicaqe poră. }\end{array}$ & $\begin{array}{l}\text { Et mon Shaban a sorti } \\
\text { un grand couteau blanc à dents, les gars! } \\
\text { D'un seul coup il a frappé mon Shaban } \\
\text { [et] il a étripé Nailitsa. }\end{array}$ \\
\hline $\begin{array}{l}\text { 3an-ta, baba, me so kerdǒm !... } \\
\text { E Altäna me mudardǒm, } \\
\text { me ćhingerdǒm! } \\
\text { Arakhlǒm la e geraleça, } \\
\text { e geraleça, e gidiaça } \\
\text { and-o jaxäri. } \\
\text { Ćumiden pes, dandelen pes } \\
\text { paś-paśes } \theta e, \text { koć-koćăâ } \\
\text { oj e geraleça! }\end{array}$ & $\begin{array}{l}\text { Sache, mon père, ce que j'ai fait !... } \\
\text { C'est moi qui ai tué Altına, } \\
\text { qui l'ai égorgée! } \\
\text { Je l'ai trouvée avec le chauve, } \\
\text { avec le chauve, avec le diable } \\
\text { dans l'étable. } \\
\text { Ils s'embrassent, ils se mordent } \\
\text { côte à côte, genou contre genou } \\
\text { elle et le chauve! }\end{array}$ \\
\hline
\end{tabular}

8 On voit ici réapparaître la figure du Turc diabolique appelé ici gidia (cf. bulg. гидийски " diabolique, etc. ») et " le chauve», en raison de divers couvre-chefs populaires chez les Turcs (de même que chez les Albanais, surnommés eux aussi «les chauves, les galeux » pour la même raison du fez / qeleshe).

9 Face à ces Turcs, violents mais aussi coureurs de jupons, usant de leur ascendant social, les Rroms dans l'ensemble paraissent partager en tout les souffrances du peuple bulgare, et ils sont même mobilisés pour défendre les frontières - peut-être contre les Turcs justement, comme dans cette chanson de recrutement d'un type très courant dans toute l'Europe mais rare chez les Rroms (pièces non citées ici, respectivement 27 et 28 dans le manuscrit de Manuś Romanov) :

\begin{tabular}{|l|l|}
\hline Aćhov devleça, daj!e, & Adieu, ma mère, \\
me 3àva manqe ka-i grànica, & je vais à la frontière, \\
ka-j grànica, kaj maren pe, & à la frontière où ils se battent, \\
ka-j grànica, kaj ćhinen pe. & à la frontière, où ils s'égorgent. \\
\hline
\end{tabular}

à moins qu'ils ne doivent prendre part à la « guerre serbe » :

\begin{tabular}{|c|c|}
\hline $\begin{array}{l}\text { O barabàno mardilă } \\
\text { ande amari pùsto malàva. } \\
\text { Maripe putardilo - } \\
\text { o sërbsko maripe. }\end{array}$ & $\begin{array}{l}\text { Le tambour a retenti } \\
\text { dans notre quartier désert. } \\
\text { La guerre a éclaté- } \\
\text { la guerre serbe. }\end{array}$ \\
\hline
\end{tabular}

10 Un grand nombre de Rroms de ce corpus portent un nom musulman (Śàza, Mamùdi, Alia, Anìfa, Sèjo, Asàni, Amèdi, Alil, Emìne, Elmàzi, Jaśàri etc. - on remarque la 
disparition des « $\mathrm{h} »)$, davantage que de prénoms slaves (Cèn[k]a, Danka, Stanka, Neda, Petrùna, Vànko, etc...). En outre, les chansons présentent non seulement un apport lexical turc très important, mais des vers entiers, souvent des expressions toutes faites mais pas seulement, montrent l'impact de la langue et de la culture turques sur ces locuteurs. Le volume de cet élément turc est en volume comparable à celui de l'élément bulgare. Bien entendu, on a les inévitables (j'écris à la turque) anem " ma mère ", canım " mon âme " (en fait souvent, "à mon avis, ma foi »), evlâdım « mon enfant ", paşam «mon pacha " etc... : «canim Emine, kizim Emine..." ... (refrain de la chanson 24). Certains parlers présentent la particule interrogative turque $m i / m u$ : « Ni ćalilan $m l$, be, Angele, kadale parne ćućorrën $\theta e$ ?» «Tu n'es pas rassasié, Angel, de ces petits seins blancs ?», « Tu $m \imath$ san mi rromnorri, Danko, mi parni dasnorri ? » «Es-tu ma petite femme, Danka, ma petite chrétienne ? » Plus surprenante est la présence d'une marque de pluriel turc à un emprunt bulgare : tärgovcolar « commerçants » (mais il est vrai que ce lar/ler final peut se cumuler au pluriel autochtone dans plusieurs langues, surtout en vérité dans les emprunts eux aussi turcs). On a également des paradigmes verbaux turcs, insérés dans la phrase rromani - phénomène connu également du parler kabuzi d'Albanie et de plusieurs de Grèce : «Benzentım tut, Fatime, upral te daja日e " «Je t'ai prise, Fatima, pour ta mère » - on remarque que la racine turque «ressembler » a été refondue sémantiquement selon le bulgare оПРИличАВА « il prend pour ». Comme dans bien des parlers, les numéraux sont empruntés : «Mo gavali o sastruno, lele, doksan oka. » « Ma flûte en fer, oh la la, celle de 45 kilos ». Dans certains cas c'est un vers entier en turc : « Bujurun aalar, bujurun bejler! " « Je vous en prie, les agas, je vous en prie les beys " - expression de courtoisie turque longtemps panbalkanique. La citation est fréquente : « Ali, zambas Ali, but keran buti, but parês lian, ellere, gündere dokäm etmedin !» « Ali, Ali maquignon, tu as beaucoup travaillé, tu as gagné beaucoup d'argent, mais on ne peut contenter tout le monde et son père ... » avec une expression turque toute faite correspondant au fameux vers de La Fontaine et présentant le statut de véritable citation. Ce phénomène est courant dans les milieux multilingues, où le passage d'une langue à l'autre, loin d'indiquer un recours au parler d'autrui pour compenser une lacune, marque la maîtrise de plusieurs registres et de plusieurs patrimoines. De même "Arekte da, dünyade da, mo phral isi, phralipe kerdam " "Tant dans ce monde que dans l'autre, c'est mon frère, nous avons scellé fraternité ». Enfin il y a parfois des répliques entières rapportées en turc, il s'agit d'alternance de codes (code-switching, qu'il serait fastidieux de citer - on en trouvera dans les chants cités).

11 La ballade rromani de Roumanie recoupe les textes de Bulgarie en ce qui concerne la description des Turcs et de leurs agissements, même si la langue employée n'a recours à pratiquement aucun mot ou expression turque, ce qui révèle évidemment une bien plus grande distance des narrateurs par rapport à la sphère ottomane que dans le cas bulgare. Il s'agit du récit d'un jeune homme qui part à Constantinople découvrir le monde mais tombe ivre au bord de la mer. C'est là que les Turcs (Xoraxaja « musulmans ») entrent en scène, le ligotent et le conduisent à l'empereur : 


\begin{tabular}{|l|l|}
\hline Ël Xoraxaja ćorăl resle & Les musulmans en cachette sont arrivés \\
thaj les zumaven te phanden, & et essayent de l'attacher, \\
K-o amperàto t'angăren. & pour le conduire à l'empereur. \\
Ëkh këtàna kaj avèla: & Un soldat qui passait par là: \\
«So kerèna, Xoraxa'!len, & "Que faites-vous, les musulmans, \\
Aman Devl!a, kaj dilàjlen? & Pitié, mon Dieu, mais vous êtes fous? \\
Tumenqe rodèna bàjo, & Vous cherchez pour vous un tracas \\
Savo nas maj and-o tràjo!» & comme vous n'en avez jamais eu de votre vie?» \\
\hline
\end{tabular}

L'autocrate le fait attacher sous un rocher pour qu'il y périsse - mais son père, alerté par un oiseau, parvient à le sauver en réduisant à l'impuissance l'empereur par une gifle magistrale. Les Turcs ne font pas ici preuve de cruauté mais plutôt de fourberie pour un intérêt mesquin, dans la mesure où ils agissent sur leur propre initiative, escomptant une récompense du souverain. Au-delà de la revanche de l'opprimé, du Rrom certes, mais plus largement aussi des Balkans sous le joug turc, par un coup à la personne et à l'honneur du plus grand des Turcs, tourné en dérision par une simple gifle, on retrouve le motif de l'oiseau messager. Alors que dans le texte de Gafur Raśidi (cf. note 2) les deux corbeaux portaient la main coupée du héros, nous avons ici un vautour qui porte une lettre, ce qui indique un niveau d'instruction plus élevé et n'est pas sans rappeler les chansons composées dans les camps de concentration nazis où le chanteur prie un oiseau de porter une lettre, pleine de souffrance, à son père ou un autre membre de son entourage :

\begin{tabular}{|c|c|}
\hline $\begin{array}{l}\text { Ax, tu kalo ćiriklorro, } \\
\text { Kalo, źalutno aj korro, } \\
\text { Manqe ligăr dur kava lil; } \\
\text { Ok' te naśti vazdes o lil } \\
\text { Kaj si pharo, pherdo dukha, } \\
\text { Atòska buxlăr te phaka }\end{array}$ & $\begin{array}{l}\text { Ah, toi, petit oiseau noir, } \\
\text { Noir, compatissant et aveugle, } \\
\text { Emporte au loin pour moi cette lettre; } \\
\text { Mais si tu ne peux soulever la lettre } \\
\text { Qui est lourde, pleine de souffrances, } \\
\text { Alors étend tes ailes. }\end{array}$ \\
\hline $\begin{array}{l}{ }^{3} \text { a me phralente aj mothov } \\
\text { So adaj si jekh baro bov, } \\
\text { So o Devel kerdilăs thuv } \\
\text { Aj kalărdăs i sasti phuv. }\end{array}$ & $\begin{array}{l}\text { Va chez mes frères et dis } \\
\text { Qu'ici il y a un grand four, } \\
\text { Que le Ciel est devenu fumée } \\
\text { Et qu'il a noirci toute la terre. }\end{array}$ \\
\hline
\end{tabular}

Pour en revenir à la ballade de Roumanie, on y relève deux particularités lexicales :

- le souverain des Turcs est appelé amperàto « empereur », titre habituellement réservé aux rois chrétiens, notamment byzantins, mais il est difficile d'expliquer - sinon par indigence lexicale ou simplification - pourquoi l'auteur n'a pas utilisé ici un turcisme : sultàno, agàvo ou autre.

- l'autre point qui intrigue est l'emploi de dorav masc. "vaste étendue d'eau, lac », terme habituellement réservé, du moins dans ce dialecte, au Danube. Toutefois il n'est pas exclu que nous ayons affaire à une attestation du sens ancien, encore présent dans les autres parlers (derăv fém. « mer »).

- Ces quelques extraits montrent combien la population rromani, à l'époque ottomane, avait fait sien le regard des autres peuples sur l'occupant et ceci n'est pas sans rappeler une attitude similaire des Boša ${ }^{8}$ d'Arménie faisant corps avec la population arménienne, comme 
le décrit l'épopée cyclique hélas perdue dans son intégrité mais dont le contenu nous est parvenu grâce au résumé de Papazian : une jeune Boša est violée au bord d'une route par un chefaillon turc tandis que son couard de mari tourne le dos; par la suite la soif de vengeance de la victime forcit d'autant plus que son mari met son servilisme et sa pleutrerie aux pieds des occupants. L'héroïne est tuée dans un combat contre ses bourreaux et ses hauts faits sont rapportés au coin du feu dans un camp; or, lorsque le narrateur termine le récit en pleurant la mort de l'héroïne, une petite fille se lève à la fin de chaque chant et déclare : « non, elle n'est pas morte, c'est moi » - ce qui, bien entendu donne le signal du départ d'un nouveau cycle. Mais déjà nous ne sommes plus dans les Balkans : le récit a pour théâtre le Caucase.

\section{Corpus de Bulgarie}

\section{CIDINDE SAS O TRIN PHRALA [MR 1]}

Rasim Aguśev, né le 7.3.1902 (Sofia, 27.10.53²)

\begin{tabular}{|c|c|}
\hline Cidinde sas o trin phrala, & Ils se sont réunis les trois frères, \\
\hline o trin phrala, o amala, & les trois frères, les amis, \\
\hline cidinde sas pe rromněnça. & ils se sont réunis avec leurs femmes. \\
\hline Lel len anglal jekh potèra, & Une potéra les surprend par devant, \\
\hline lel len palal jekh potèra, & une potéra les surprend par derrière, \\
\hline aćhile maśkar o trin phrala. & ils sont restés au milieu les trois frères. \\
\hline O bareder phral phenèlas: & Le frère aîné déclarait : \\
\hline «Aj, phrala!len, amala!len, & «Ah, mes frères, mes amis, \\
\hline ćhiven piro, den naśiba, & jouez des jambes, prenez la fuite, \\
\hline te uxtavas amaro śero & pour que nous sauvions notre tête, \\
\hline Xoraxan $\theta a r$, segmeněn $\theta a r ! »$ & des Turcs, des segmènes!» \\
\hline O cikneder phral phenèlas: & Le frère cadet disait: \\
\hline «Aj, phrala!len, amala!len, & "Ah, mes frères, mes amis, \\
\hline me na naśav potera $\theta a r$ & je ne fuis pas la potéra, \\
\hline la3 isi man me rromna $\theta a r$, & j'aurais honte devant ma femme, \\
\hline 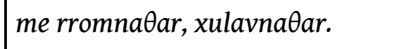 & devant ma femme, ma maîtresse de maison. \\
\hline Aj, rromni!e, xulavni!e, & Ah ma femme, ma maîtresse de maison, \\
\hline de ta man $\theta$ e mi xarbica, & donne-moi donc mon javelot, \\
\hline tu-da le-ta mi makravli, & et toi aussi prends donc gourdin, \\
\hline thaj te terdos palal man $\theta e$, & et tiens-toi derrière moi \\
\hline te na ćalavel man dekh Xoraxaj!» & que ne me frappe pas quelque Turc!» \\
\hline Tele dinas, upre dinas & Il a frappé par en bas, par en haut, \\
\hline rat gazindas $3 i$ koća $\theta e$ & Il a foulé le sang jusqu'au genou, \\
\hline ćhingerdas len $3 i$ jekhes $\theta e$. & il les a abattus jusqu'au dernier. \\
\hline Sade mukhlas bulük-baśis, & Il n'a épargné que le capitaine, \\
\hline bulük-baśis, e vojvodas & le capitaine, le voiévode \\
\hline «Ade, mança te xas, te pias » & "Allez suis-moi, allons manger allons boire». \\
\hline
\end{tabular}

\section{AIDE, ŚAZO, ŚAZO [MR 3]}

Sali Ne3ipov Ćobanov, 1919 (Samokov, 1954) 


\begin{tabular}{|c|c|}
\hline $\begin{array}{l}\text { Ajde, Śazo, Śazo, } \\
\text { k-i loli mehàna, (2) } \\
\text { k-i 3amläz odàja. }\end{array}$ & $\begin{array}{l}\text { Allons Shaza, Shaza, } \\
\text { à la taverne rouge, } \\
\text { à la chambre vitrée. }\end{array}$ \\
\hline $\begin{array}{l}\text { Me na 3àvas, Mamud, } \\
\text { k-i bari mehàna, (2) } \\
\text { k-i 3amläz odàja. } \\
\text { Othe isi, Mamud, } \\
\text { bare Xoraxaja. } \\
\text { Tut ka maren, Mamud, } \\
\text { mane man on ka len. }\end{array}$ & $\begin{array}{l}\text { Je ne vais pas Mamoud, } \\
\text { à la taverne rouge, } \\
\text { à la chambre vitrée. } \\
\text { Là-bas il y a Mamoud, } \\
\text { des notables turcs. } \\
\text { Toi ils vont te frapper } \\
\text { moi ils vont me prendre. }\end{array}$ \\
\hline $\begin{array}{l}\text { Ajde, mança, Śazo, } \\
\text { k-i bari mehàna, (2) } \\
\text { k-i 3amläz odàja. }\end{array}$ & $\begin{array}{l}\text { Allez, viens avec moi Shaza, } \\
\text { à la taverne rouge, } \\
\text { à la chambre vitrée. }\end{array}$ \\
\hline $\begin{array}{l}\text { Te lav tuqe, Śazo, } \\
\text { śel dràma rakia; } \\
\text { tu te ćhores, Śazo, } \\
\text { Me te piav-o Mamud. }\end{array}$ & $\begin{array}{l}\text { Que je prenne pour toi, Shaza, } \\
\text { cent drams [Cent drams d'eau-de-vie } \\
=\text { environ } 310 \text { grammes.] d'eau de vie. } \\
\text { Que toi verses, Shaza, } \\
\text { Et moi Mamoud que je boive }\end{array}$ \\
\hline $\begin{array}{l}\text { Uśti othar, Śazo, } \\
\text { beś-ta mamuj man } \theta e, \\
\text { krafla te ćhinàvas, } \\
\text { lele, gurvorrenqe. }\end{array}$ & $\begin{array}{l}\text { Lève-toi de là, Shaza, } \\
\text { Assieds-toi donc en face de moi, } \\
\text { que je coupe des clous de fer à cheval, } \\
\text { oh la la, pour les bœeufs. }\end{array}$ \\
\hline $\begin{array}{l}\text { Phude manqe, Śazo, } \\
\text { te taton me fela, } \\
\text { te del vrasi fela. } \\
\text { Eke kotar avlas } \\
\text { o lub o Re3èpi, } \\
\text { pe parne grasteça, } \\
\text { pe parne ćhuraça. }\end{array}$ & $\begin{array}{l}\text { Souffle, Shaza, souffle, } \\
\text { que chauffent mes tranches } \\
\text { [de fer], } \\
\text { qu'elles chauffent à blanc. } \\
\text { Et voilà que vient } \\
\text { Redjep le débauché, } \\
\text { avec son cheval blanc, } \\
\text { avec son sabre étincelant. }\end{array}$ \\
\hline $\begin{array}{l}\text { Uśti othar, Śazo, } \\
3 a \text { tuqe androrre, } \\
\text { te uras te dibèka [ipèka] } \\
\text { te neve śalvàra. }\end{array}$ & $\begin{array}{l}\text { Lève-toi de là, Shaza, } \\
\text { rentre à la maison, } \\
\text { enfile tes soieries } \\
\text { tes chalevares neuves. }\end{array}$ \\
\hline $\begin{array}{l}\text { 3ikaj te vakerel, } \\
\text { ikaldas o fela, } \\
\text { o lub, o Re3èpi } \\
\text { ikaldas parni ćhuri }\end{array}$ & $\begin{array}{l}\text { Tandis qu'il parlait, } \\
\text { il retira ses fers rouges, } \\
\text { et Redjep le débauché } \\
\text { tira son sabre clair. }\end{array}$ \\
\hline $\begin{array}{l}\text { Inklini i Śaza, } \\
\text { dikhel anglal vudar- } \\
\text { ov pe parne ćhuraça, } \\
\text { ov pe tate felěnça. }\end{array}$ & $\begin{array}{l}\text { Voilà Sheza qui sort } \\
\text { elle regarde devant la porte- } \\
\text { l'un avec son épée blanche, } \\
\text { l'autre avec ses fers ardents. }\end{array}$ \\
\hline
\end{tabular}




\begin{tabular}{|l|l|}
\hline $\begin{array}{l}\text { Dinas piśta Śaza, } \\
\text { lele, pe rromesqe: } \\
\text { "Jeter, Mamud, jeter, } \\
\text { jeter, mudardan les!» }\end{array}$ & $\begin{array}{l}\text { Shaza a poussé un cri, } \\
\text { oh la la, à son mari v: } \\
\text { "Arrête, Mamoud, arrête, } \\
\text { ça suffit, tu vas le tuer!» }\end{array}$ \\
\hline $\begin{array}{l}\text { "Korkma, Śazo, korkma, } \\
\text { me les ka mudarav. } \\
\text { Te na phirel, mori, } \\
\text { palal tüe, Śazo. }\end{array}$ & $\begin{array}{l}\text { "Ne crains rien, Shaza, } \\
\text { je m'en vais le tuer } \\
\text { qu'il ne vienne pas, ma mie, } \\
\text { te poursuivre Shaza. }\end{array}$ \\
\hline $\begin{array}{l}\text { Ajde, mança, Śazo, } \\
\text { k-i loli mehàna, } \\
\text { te lav tuqe, Śazo, } \\
\text { śel dràma rakia. " }\end{array}$ & $\begin{array}{l}\text { Allons Shaza, Shaza, } \\
\text { à la taverne rouge, } \\
\text { que je commande pour toi, } \\
\text { Shaza, cent drams d'eau-de-vie.» }\end{array}$ \\
\hline
\end{tabular}

\section{PHUTER MANQE VUDARORRO [MR 4]}

Demka Cvetanova, née en 1910 (Sofia, 17.11.1953)

\begin{tabular}{|c|c|}
\hline $\begin{array}{l}\text { "Aj, rromnile, xulanile, } \\
\text { phuter manqe vudarorro, (2) } \\
\text { balval lilas mo dumorro." }\end{array}$ & $\begin{array}{l}\text { "Ah ma femme, maîtresse de maison, } \\
\text { ouvre-moi la porte, } \\
\text { le vent m'a paralyséle dos." }\end{array}$ \\
\hline $\begin{array}{l}\text { "Me na phutrav vudarorro, } \\
\text { mo rrom gelo biaves } \theta e, \\
\text { biaves } \theta \text { e Devaněn } \theta e . »\end{array}$ & $\begin{array}{l}\text { "Je ne t'ouvre pas la porte, } \\
\text { mon mari est parti à une noce, } \\
\text { à une noce à Devyane." }\end{array}$ \\
\hline $\begin{array}{l}\text { « Aj, rromnile, xulanile, } \\
\text { balval lilas mo dumorro, (2) } \\
\text { phuter manqe vudarorro!» }\end{array}$ & $\begin{array}{l}\text { "Ah ma femme, maîtresse de maison, } \\
\text { le vent m'a paralysé le dos, } \\
\text { ouvre-moi la porte." }\end{array}$ \\
\hline $\begin{array}{l}\text { «Sikter burdan, köpek oolu! } \\
\text { Tu na sinan mo rromorro.» }\end{array}$ & $\begin{array}{l}\text { "F... le camp, fils de chien! } \\
\text { Tu n'es pas mon cher mari. » }\end{array}$ \\
\hline $\begin{array}{l}\text { "Sigo phuter, xulanile, } \\
\text { sar na sinom to rromorro?" }\end{array}$ & $\begin{array}{l}\text { "Ouvre vite, maitresse de maison, } \\
\text { comment ne serais-je pas ton cher mari? " }\end{array}$ \\
\hline $\begin{array}{l}\text { "Ćhiv to śero pen3era } \theta \text { ar, } \\
\text { me te dikhav tu li sinan!" }\end{array}$ & $\begin{array}{l}\text { "Passe la tête par la fenêtre, } \\
\text { que je puise voir si c'est toi!" }\end{array}$ \\
\hline $\begin{array}{l}\text { Ćhivtas śero pen3era } \theta a r, \\
\text { te dikhel les i rromnorri. }\end{array}$ & $\begin{array}{l}\text { Il a passé la tête par la fenêtre } \\
\text { pour que sa chère femme le voie. }\end{array}$ \\
\hline $\begin{array}{l}\text { "Sikter burdan, köpek oolu!»- } \\
\text { thaj lilas o toverorro } \\
\text { ta ćhindasas i menorri, } \\
\text { a o trùpos avri aćhlo. }\end{array}$ & $\begin{array}{l}\text { "F... le camp, fils de chien!" } \\
\text { et elle a pris la hachette } \\
\text { et lui a coupé le cou } \\
\text { et le corps est resté dehors. }\end{array}$ \\
\hline
\end{tabular}




\begin{tabular}{|l|l|}
\hline $\begin{array}{l}\text { Ćhingerdas les kotoren } \theta e, \\
\text { ingaldas les veśorres } \theta e,(2) \\
\text { umblaldas les kaś kaśtes } \theta e .\end{array}$ & $\begin{array}{l}\text { Elle l'a découpé en petits morceaux, } \\
\text { elle l'a emporté dans la forêt, } \\
\text { et a tout pendu d'arbre en arbre. }\end{array}$ \\
\hline $\begin{array}{l}\text { Umblaldas les kaś kaśtes } \theta e \\
\text { thaj piśindas i rromnorri: } \\
\text { «ЕЛАТЕ, ЧАВКИ, ЕЛАТЕ, } \\
\text { МЯСО ИМА ЕФТИнИЯ!» }\end{array}$ & $\begin{array}{l}\text { Elle l'a pendu d'arbre en arbre } \\
\text { et elle a écrit la chère épouse: } \\
\text { «Venez, les corbeaux, venez, } \\
\text { il y a de la viande à pas cher!» }\end{array}$ \\
\hline
\end{tabular}

\section{CENO, LE [MR 5]}

Rasim Aguśev, né le 07.03.1902 (Sofia, 27.10.53)

\begin{tabular}{|c|c|}
\hline $\begin{array}{l}\text { A ćhe, Ceno, tu ćhe, Ceno, } \\
\text { le to bùklos, 3a paněsqe, }\end{array}$ & $\begin{array}{l}\text { À Tsena, ma fille, Tsena, } \\
\text { prends ta gourde en bois, va chercher de l'eau, }\end{array}$ \\
\hline $\begin{array}{l}\text { Ceno, le! } \\
\text { Ta an manqe śudro pani, } \\
\text { śudro pani xaingaqo. }\end{array}$ & $\begin{array}{l}\text { Tsena, ah! } \\
\text { Et apporte-moi de l'eau froide, } \\
\text { de l'eau froide de la source, }\end{array}$ \\
\hline $\begin{array}{l}\text { Ceno, le! } \\
\text { Tu te ćhores, me te piav, } \\
\text { me te piav te vastes } \theta \text { ar, }\end{array}$ & $\begin{array}{l}\text { Tsena, ah! } \\
\text { Que toi tu verses, moi que je boive, } \\
\text { moi que je boive de ta main, }\end{array}$ \\
\hline $\begin{array}{l}\text { Ceno, le! } \\
\text { me te piav te vastes } \theta \text { ar, } \\
\text { te vastes } \theta \text { ar ćaćunes } \theta a r,\end{array}$ & $\begin{array}{l}\text { Tsena, ah! } \\
\text { moi que je boive de ta main, } \\
\text { de ta propre main à toi, }\end{array}$ \\
\hline $\begin{array}{l}\text { Ceno, le! } \\
\text { Aj, geli sas pajorresqe, } \\
\text { pajorresqe xaingaqo, }\end{array}$ & $\begin{array}{l}\text { Tsena, ah! } \\
\text { Et elle est allée chercher de l'eau } \\
\text { de l'eau de la source, }\end{array}$ \\
\hline $\begin{array}{l}\text { Ceno, le! } \\
\text { Thaj resle la biś Xoraxaja, } \\
\text { biś Xoraxaja, sa ćhurěnça, }\end{array}$ & $\begin{array}{l}\text { Tsena, ah! } \\
\text { Et sont arrivés vingt Turcs, } \\
\text { vingt Turcs, tous armés }\end{array}$ \\
\hline $\begin{array}{l}\text { Ceno, le! } \\
\text { Dolde bùklos thaj phagle les. } \\
\text { Mudarde la, chingarde la. } \\
\text { Ceno, le! }\end{array}$ & $\begin{array}{l}\text { Tsena, ah! } \\
\text { Ils ont pris la gourde l'ont cassée. } \\
\text { Ils ont tuée Tsena à coups de sabre, } \\
\text { Tsena, ah! }\end{array}$ \\
\hline
\end{tabular}

\section{UKÈSTILO PE KALE ARAPĚS [MR 6]}

Najla Alieva, née en 1885 (Sofia, 01.10.1953) 


\begin{tabular}{|c|c|}
\hline 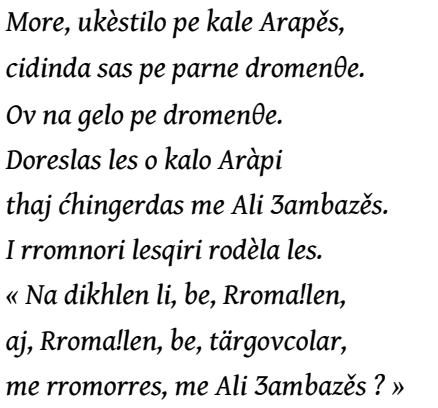 & $\begin{array}{l}\text { Les gars, il a enfourché son Aràpi noir } \\
\text { et s'en est allé par les vastes routes. } \\
\text { Il n'a pas pu suivre sa route, } \\
\text { le Nègre esclave l'a rattrapé } \\
\text { et il a égorgé mon maquignon Ali. } \\
\text { Sa petite femme part à sa recherche. } \\
\text { "Vous ne l'avez pas vu, les Rroms, } \\
\text { eh, les Rroms, les gars, les commerçants, } \\
\text { mon petit mari, mon maquignon Ali? " }\end{array}$ \\
\hline$\ldots \ldots \ldots$ & .......... \\
\hline $\begin{array}{l}\text { Devl!a, o pirrorre and-o zengin aćhle. } \\
\text { O śerorro - jekh rigorri gelo. } \\
\text { A ćhe, nine, 3andan, mi dajorri! } \\
\text { Lilas o śero pe angaläe. } \\
\text { "Aj, Ali, be, phuter te jakhorra! } \\
\text { Aj, Ali, be, 3andan, mo rromorro!" } \\
\text { "More, jekh dies, duj dies } \\
\text { te rodes man, borile, ternile, } \\
\text { trine kurkenqirile, rakli!e. } \\
\text { Aćhon tuqe pan3 ćorrorre, } \\
\text { pan3 ćorrorre, sa murśorre ćhave. } \\
\text { Me ćorrorre, me korkorre, de!» }\end{array}$ & $\begin{array}{l}\text { Mon Dieu, les pieds sont restés dans l'étrier. } \\
\text { La tête - elle a roulé sur le côté. } \\
\text { Ah, ma mère, de tout cour, ma petite mère! } \\
\text { Elle a pris la tête dans ses bras. } \\
\text { «Ah, Ali, ouvre tes yeux! } \\
\text { Ah, Ali, de tout cour mon petit mari!» } \\
\text { "Tu peux bien me chercher un jour, deux jours, } \\
\text { me chercher jeune épouse, } \\
\text { jeune Bulgare de trois semaines. } \\
\text { Je te laisse cinq orphelins, } \\
\text { cinq orphelins, tous des garçons. } \\
\text { Mes pauvrets, mes esseulés!» }\end{array}$ \\
\hline
\end{tabular}

\section{E ALI 3AMBAZESQO SUNO [MR 9]}

Ivan Dimitrov Kräcov, né en 1896 (Sliven, 05.01.1954) 
- Ajrat dikhêm ekh sunorrô :

mä śerës $\theta$ e lolô jazmas,

mä pirënde kalu ćizmas.

Motha manqe, anäm, motha manqe, canım,

so vakerla morrô sunorrô ?

- A be, Ali, ${ }_{3}$ ambaz Ali,

evlâdım, kızınım,

Khainês vakèrla to sunorrô:

ô lolô jazmas - xaberìci,

ô kalốcizmas, vaj - limorìci.

Loize tut, Ali3ës, loize tut,

käkäle bare ga3ên $\theta a r$,

ći ola gonizèna tut,

aśal ti rromnaqi Gülsujeqi !

- Źanki an-ta ma sinia cavala,

tê ura, tê3anka dromorës $\theta e$,

te mi parni kucik tê kucira la,

tê andrë tê thova sultanësqô thuv.

Me mä 3anka Zaara panairës $\theta$ e.

Uśtilô, uras pês, cizias.

Gelo tar $3 i$ Ćirpanësqê ćairën $\theta \hat{e}$.

Dikhèla, telal avèna

trin 3ene, trin bare ga3ê,

ô trin-da grastënça.

Ta vakärna lësqë :

- Miśto ajan, 3ambaz Ali!

Kariga cizian käkäle vakäcês $\theta \hat{e}$ ?

Ta vakèrla ô $3 a m b a z$ Ali :

- Miśto arakhen, aalar, bejler?!

Me 3ankava Zaara panairën $\theta \hat{e}$.

Bujurun, aalar, bujurun, bejler,

mëre sultanësqô thuves $\theta$ ar

të pacarën po jekh thuvali !

- Amê na manga terê thuves $\theta$ ar.

Amê mangàsa torô gudlo ozi !

Banzardê lêskô laćhô śorô

ta ćhindê lês, ta ćhitê lês

ekhe riga $\theta \hat{e}$ - ortuni rig

palê ô léśi mukhlê

uprë dromorrës $\theta \hat{e}$.

Tê nakhistê - gelê!

Telal avila ô Dinkô ćorba3is.

oda-da 3àla Zaara panairës $\theta \hat{\text {. }}$

Dikhla opr-ô dromorrô

chipas kaila.

Tê ovêl bur? - nanäj bur.

Tê ovêl 3ukel ? - nanäj 3ukel.

So si käka kô kaila?

Ta gelo tê dikhel tar-

lèsi bi śerësqô.

Katar dikhlas, kotar dikhlas

tê alakhêl ô śerorrô -

ek da alakhala

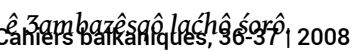

Ta mukhêla lês $3 i$ leśes $\theta \hat{e}$.

Irizèla palê papalê and-ô gav,

ta rivizèla duraldan

e 3ambazêsqê puräj däjreqê :

- Cette nuit j'ai eu un songe :

Sur ma tête un fichu rouge

A mes pieds, des bottes noires.

Dis-moi, ma mère, dis-moi petite mère,

Que signifie mon songe?

- Ah mon gars, mon Ali, mon maquignon

Mon enfant, mon petit,

Ton songe ne dit rien de bon.

Le fichu rouge, c'est une nouvelle,

Les bottes noires, c'est une tombe.

Garde-toi, protège-toi,

De ces grands Turcs qui te pourchassent

À cause de ta femme, Gulsui.

- Allez me chercher ma grande cape noire,

Que je la revête et que je prenne la route

Et ma ceinture blanche que je la ceigne

Et que je mette dans la poche du tabac de sultan

J'irai ainsi à la foire de Zaara.

Il s'habille, il met sa ceinture,

Il est allé jusqu'aux prairies de Chirpané.

Mais voilà que devant lui

s'avancent trois personnes

Trois Turcs.

Tous les trois chevauchant des pur-sang.

Et voilà qu'ils lui disent :

- Bienvenue Ali le maquignon,

Où vas-tu à une heure si matinale?

Et voilà qu'Ali le maquignon leur répond:

- Bienvenue les beys,

c'est à la foire de Zaara que je me rends,

Servez-vous mes seigneurs

de mon tabac de sultan,

Et roulez-vous une cigarette.

- Mais nous ne voulons pas de ton tabac,

Mais nous voulons ta vie, qui t'est si chère.

Ils ont coupé sa belle tête, ils l'ont décapité.

Ils l'ont jeté d'un côté de la route, du côté droit,

Et puis son corps ils l'ont laissé sur la route,

sur le pavé,

Et les voilà partis.

Voilà que vient à passer Dinko le commerçant,

Lui aussi va à la foire de Zaara,

Et il a vu sur la route quelque chose

qui fait une tache:

Peut-être un fagot?

Ce n'est pas un fagot.

Peut-être un chien?

Ce n'est pas un chien.

Qu'est-ce qui fait cette tache?

Il s'est approché, il a vu un corps sans tête

Il a regardé par ci, il a regardé par là, pour trouver la tête.

Il l'a bien trouvée.

La belle tête du maquignon.

Et il l'a laissée près du corps.

Il a rebroussé chemin jusqu'au village 


\section{MA DARA, SEJO [MR 10]}

Tair Aliev Pantenturi, né en 1902 (Samokov, 30.10.1953)

\begin{tabular}{|c|c|}
\hline 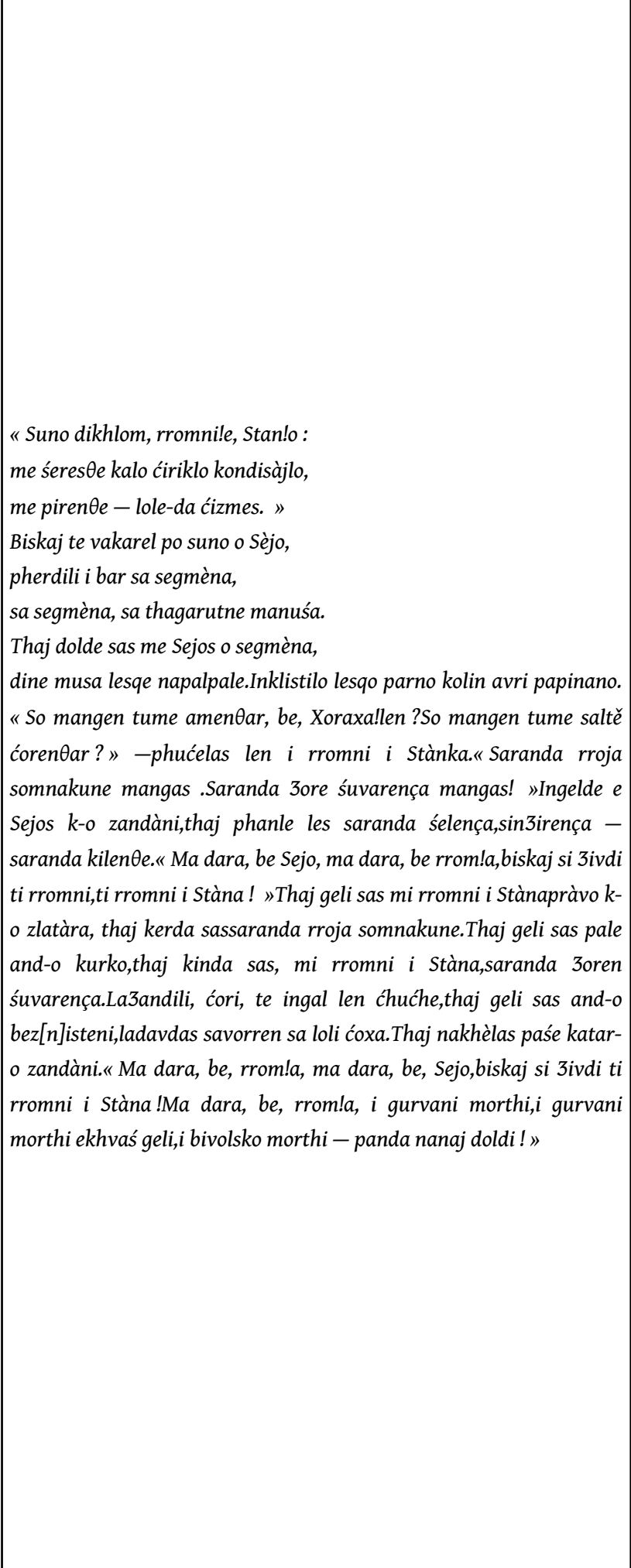 & 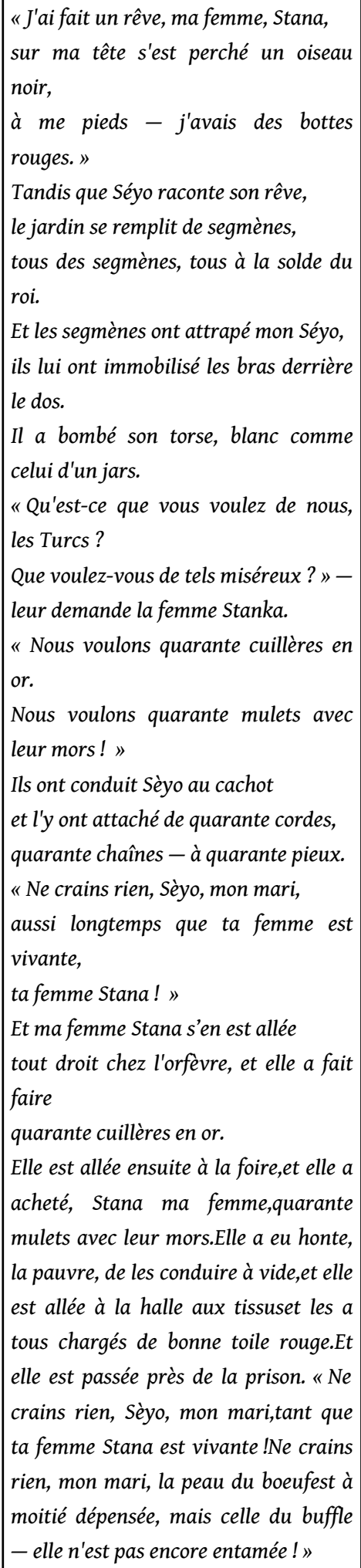 \\
\hline
\end{tabular}




\section{MI ĆHAJORRI A3AMIJKA [MR 18]}

Xüseni Aliev Bilǎlov, né le 18.05.1898 (Sofia, 19.11.1975)

«Dikh-ta, mi ćhajorri,ko marel k-o vudar,dajkha geći dòba,lele, Done, a3amijko.Te si dekh Rromorro,vudar phuter lesqe,te si dekh Ćitàki,o vudara phande,lele, mi ćhajorri!Ćitàki si beng. Rakia ka mangel.Mezesqe ka pućhel,xaborre ka ròdel,buxlaribe ka mangel.Kate ka arakhav dajkha geći dòba?"

"Ma dara, che daj!e,

lele, puranile.

Merre xurde asves

rakì ka oven.

Merre lole ćhama

mezesqe ka oven.

Merre gudle vuśta

xaborre ka oven.

Merri ući snàga

buxlaribe ka ovel.

Merre kale bala

ucharibe ka oven,

lele, daj!e puranile!»
"Regarde voir, ma fille,qui frappe à la porteà cette heure tardive,seigneur! Dona, ma candide, Si c'est quelque pauvre Rrom,ouvre-lui la porte,si c'est un Turc,ferme-lui la porte,seigneur ma fillette!Le Turc est un démon :il exigera de l'eau-de-vie.Il demandera où sont les hors-d'œeuvre,il cherchera à manger,

il voudra se coucher à l'aise.

Où trouverai-je [tout cela]

à une heure si tardive?"

"N'aie pas peur, ma mère,

ma pauvre vieille!

Mes fines larmes

se feront eau-de-vie.

Mes joues rouges

se feront hors-d'œuvre.

Mes douces lèvres

se feront plat de roi.

Mon corps svelte

se fera couche.

Mes cheveux noirs

se feront couverture

[«Couverture » au double sens

de « literie » et de « terre

qui recouvre un mort ».],

ma pauvre vieille mère.»

\section{BINE, ŚAZO [MR 19]}

Sali Ne3ipov Ćobanov, 1919 (Samokov, 1954) 


\begin{tabular}{|c|c|}
\hline 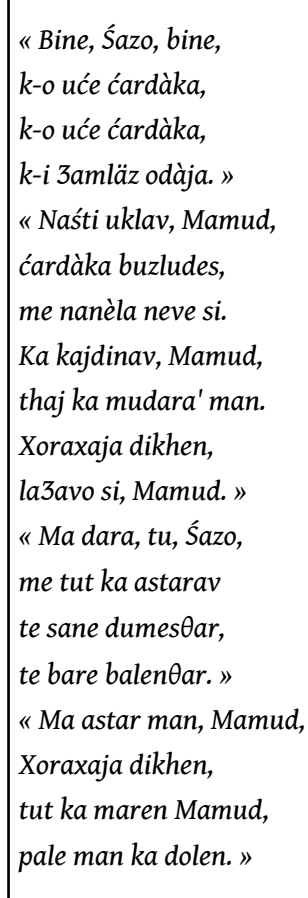 & $\begin{array}{l}\text { "Monte, Shaza, monte, } \\
\text { sur le long tchardak, } \\
\text { la terrasse de bois couverte } \\
\text { de vignes en espaliers } \\
\text { à la chambre vitrée." } \\
\text { "Je ne peux pas monter, Mamoud, } \\
\text { le tchardak est couvert de glace, } \\
\text { mes sabots sont neufs. } \\
\text { Je vais glisser, Mamoud, } \\
\text { et je vais me tuer. } \\
\text { Des Turcs regardent } \\
\text { j'aurais honte, Mamoud.» } \\
\text { "N'aie pas peur, Shaza, } \\
\text { je te retiendrai } \\
\text { par ta taille mince, } \\
\text { par tes longs cheveux.» } \\
\text { « Ne me retiens pas, Mamoud, } \\
\text { des Turcs regardent, } \\
\text { ils vont te battre Mamoud, } \\
\text { et ensuite se saisiront de moi. » }\end{array}$ \\
\hline
\end{tabular}

\section{TELAL AVÈNA, ALEMO [MR 85]}

Aśim Barămov, né en 1893 (Vidin, 16.12.1953) 


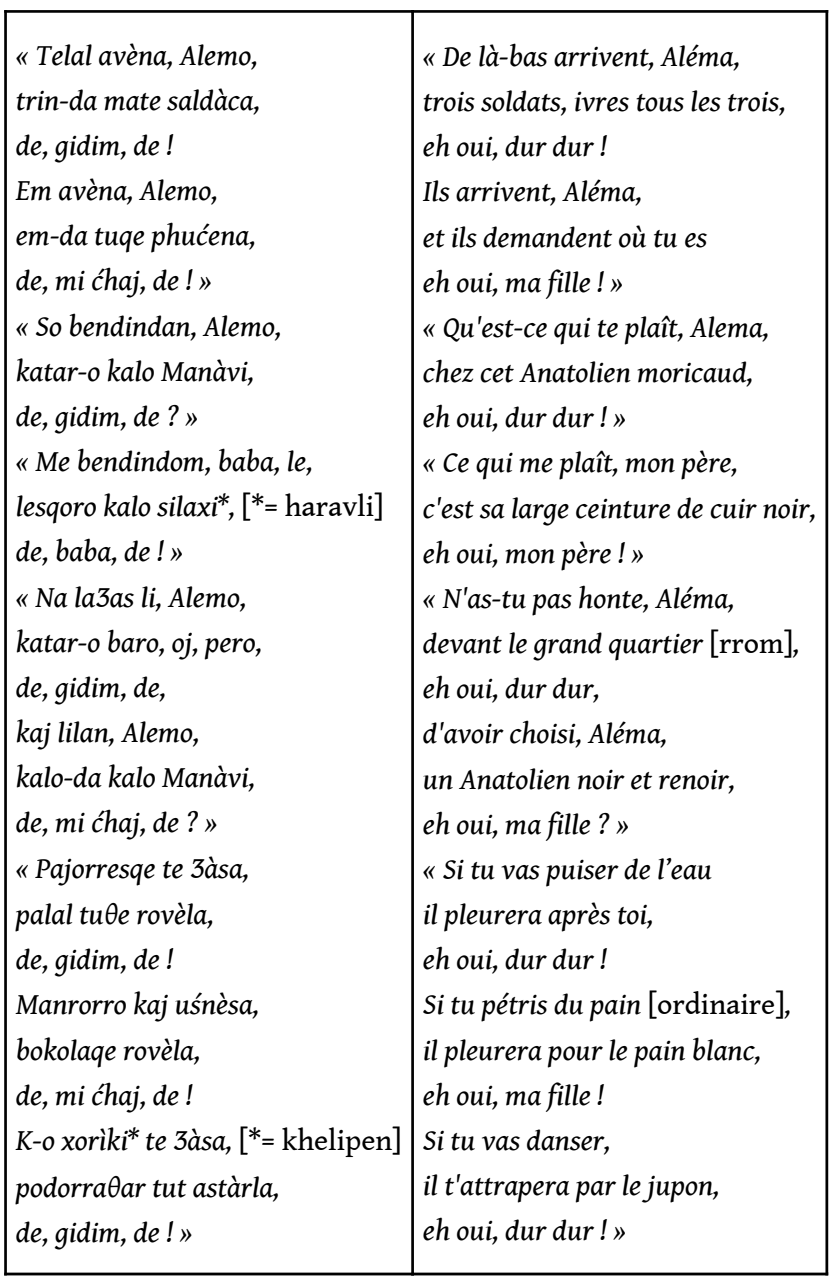

\section{MI DAJ, MO DAD VAKERE [MR 88]}

Mika Derviśeva Alkova, née en 1912 (Pirot, 19.12.1953) 
Mi daj, mo dad vakere,

phivle rrome te na lav, (2)

do ćhavenqoro dadorro.

Ale, ale, 3ino, 3ino, ćha,

ale, ale, ale, mi ćhaj ćha!

Xoraxani cikni ćhajorri,

dasikani bari bororri!

Do ćhavenqo dadorro,

do rromněnqo rromorro,

mi ergeni te lav,

mo bevorro te kerel.

Ale, ale, 3ino...

Ami ergeni te lav,

te kerel mo bevorro, (2)

te asal mo kherorro.

Ale, ale, 3ino...

A va, dad!e, tu dad!e,

ci me kam 3av k-i ćeśme,

śudro pani te anav.

3in-gär-laka, 3ino, 3ino, le, ale, ale, ale, mi rakli,

3in-gär-daka, 3ino, 3ino, ćha,

ale, ale, 3ino, mi bakri!
Ma mère, mon père [ $\left.\mathrm{m}^{\prime}\right]$ ont dit,

de ne pas épouser un veuf,

père de deux enfants.

Viens, viens, Djina, ma fille,

viens, viens, viens, ma fille!

Petite fillette turque,

grande bru chrétienne!

Père de deux enfants,

homme de deux femmes,

mais de prendre un célibataire,

pour qu'il fasse mes noces.

Viens, viens, Djina...

Mais de prendre un célibataire

pour qu'il fasse mes noces,

que ma maisonnée se réjouisse.

Viens, viens, Djina...

Ah oui, père, toi mon père,

je vais aller à la fontaine,

pour apporter de l'eau froide.

Djin-gër-laka, Djina...

Viens, viens, ma fille...

Djin-gër-laka, Djina...

Viens, viens, ma brebis... 
Chant de Roumanie - fragment (communiqué par Petre Copoiu, de Giurgiu): 


\begin{tabular}{|c|c|}
\hline 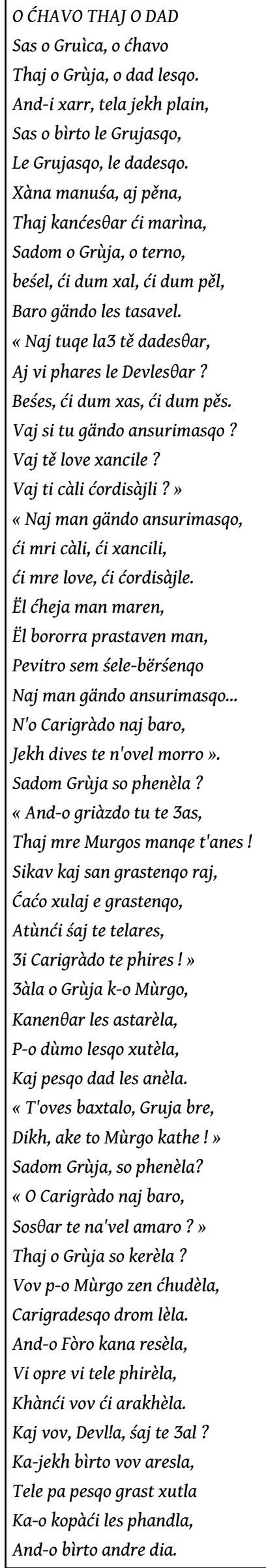 & 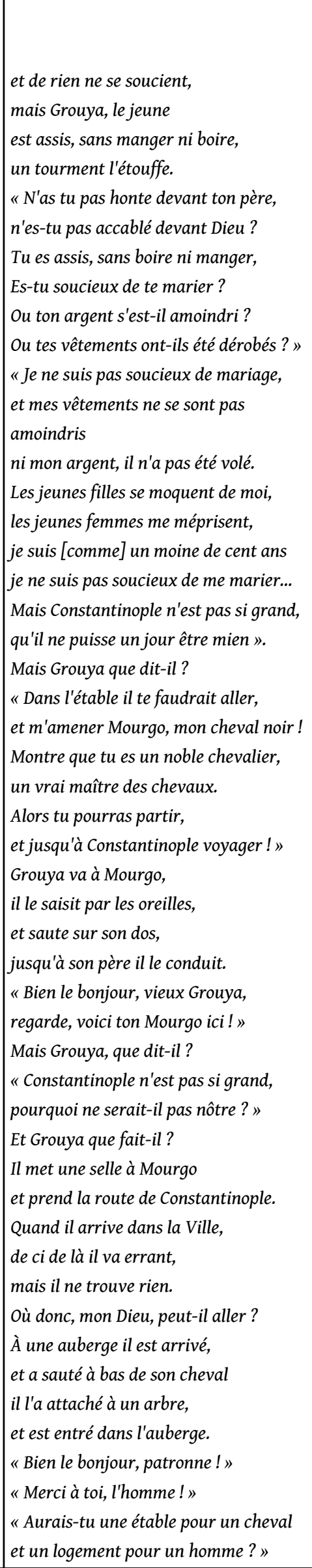 \\
\hline $\begin{array}{l}\text { "Baxt sastimos, birteśico » } \\
\text { Cahiers balkaniques, 36-37||2008 } \\
\text { " Nais tuqe, manuśela! " } \\
\text { "Si tu griàzdo pe-kh grast, } \\
\text { Haj jekh konàko pe-kh manuś? ? } \\
\text { "Si man griàzdo pe-kh grast, }\end{array}$ & $\begin{array}{l}\text { "J'ai une étable pour un cheval } \\
\text { et un logement pour un homme." } \\
\text { Qui était cette aubergiste? } \\
\text { C'était la vieille Krćmaritza, } \\
\text { une connaissance de son père, }\end{array}$ \\
\hline
\end{tabular}




\section{BIBLIOGRAPHIE}

Copoiou, Petre (env. 1970), Culegere de texte Ţiganeşte (ms.) - Giurgiu, s. d.

Courthiade, Marcel (1985), "Between oral and written textuality: the lila of the young Rromani poets in Kosovia" dans Lacio drom international n $6 / 1985$, pp. 2-20.

Ilčev, Stefan (sous la réd. de) (1974), РЕЧНИК НА РЕДКИ, ОСТАРЕЛИ И ДИАЛЕКТНИ ДУМИ В ЛИТЕРАТУРАТА НИ ОТ ХІХ И ХХ ВЕК. Sofia : Académie bulgare des Sciences.

Papazian, V. M.( 1901), Hay-Poshaner, étude ethnographique, Tiflis, 1899 (en arménien, trad. en russe et publié comme АРМЕНСКИЕ БОША (ЦЫГАНЕ): ЕТНОГРАФИЧЕСКИЙ ОЧЕРК. Moscou.

Romanov, Manuś - РОМАНЕ ПХУРЕ-ПУРАНЕ ГИЛЯ (ms.) - Sofia, décembre 1975.

\section{NOTES}

1. Une exception notable - mais ponctuelle est la radio turque de Prizren qui héberge une émission quotidienne en rromani, dirigée par le journaliste et poète Kujtim Paćaku, lui-même bon turcophone.

2. Luka Šteković, Rečnik rromskih pisaca Srbije i Crne Gore, sous presse.

3. La seule exception est un texte en vers de Gafur Raśidi Meribe e diaqo (La mort de la mère), publié dans "Between oral and written textuality: the lila of the young Rromani poets in Kosovia" dans Lacio drom international $n^{\circ} 6 / 1985, p p .2-20$, Rome. Encore le texte d'une soixantaine de vers ne mentionne-t-il pas explicitement les Turcs, mais rapporte comment la mère de Vehbi et de ses neuf frères s'effondre foudroyée lorsqu'elle découvre la main coupée de son fils apportée du champ de bataille par deux corbeaux. Fait significatif, on ne peut identifier si Vehbi et ses neuf frères se battaient du côté de Murat ou de celui de Lazare.

4. De confession musulmane, il a porté le nom «turc» de Demir Aliev jusqu'aux années 80 où l'état civil l'a mis en demeure de choisir un nom purement bulgare - ce qu'il a fait en se faisant appeler non sans humour Manuś Romanov... (Manuś est « humain » en rromani, et le clin d'œil de Romano[v] est transparent...). Les textes cités ici sont tirés d'un recueil tapé à la machine qu'il m'a confié en 1988 pour publication.

5. Les Rroms de la péninsule ibérique, appelés Gitans, ont perdu l'usage normal du rromani en raison des persécutions et se sont mis très tôt à parler la langue locale à leurs enfants ; ces derniers toutefois, en entrant dans le monde des adultes et du travail, glanaient quelques lexèmes auprès de leurs aînés, qui continuaient à parler rromani entre eux, et les utilisaient comme une sorte d'argot à fonction aujourd'hui surtout symbolique et identitaire appelée chipi calí («langue noire ») ou simplement caló. De nos jours, quelques douzaines de lexèmes sont d'usage courant mais on connaît les formes antérieures, un peu plus riches, grâce à quelques lexiques (en partie fantaisistes toutefois), des prières traduites et deux cents proverbes environ.

6. Pour ce mot $v$. infra.

7. Le mot çëtëk existe en albanais cossovar dans le sens de «enfant attardé mentalement » et une filiation n'est pas exclue avec tc. çitik.

8. Les Boša (en Turc Poşa) sont une population appelée «tsiganes arméniens » dans le Caucase et que l'on a longtemps considérée comme une branche très anciennement détachée du peuple rrom, sans doute avant même la traversée de la Perse, comme l'a relevé Ian Hancock de 
l'Université d'Austin (Texas). Ce même chercheur en est venu récemment à mettre en doute toute parenté entre ces Boša, qui s'appellent eux-mêmes Lomavren, et les Rroms, sans toutefois parvenir à une position définitive.

9. Entre parenthèses, lieu et date du collectage.

\section{RÉSUMÉS}

La publication bilingue de 11 chansons rromanies de Bulgarie et une de Roumanie, une étude des noms, locutions, expressions turques utilisées dans les chants Rroms illustre la vision rromanie du personnage du Turc. En raison de l'accès tardif des Rroms à la littérature écrite (après la Seconde Guerre mondiale dans les Balkans), il n'y a que les récits oraux qui puissent apporter du matériel pour illustrer la recherche sur tel ou tel personnage dans leur vision du monde. Après avoir expliqué les différents noms attribués aux Turcs dans les chansons populaires, cette présentation tente de définir les traits principaux de ce personnage tel qu'il se dégage de ces chansons, à savoir, impitoyable, féroce et même sadique quand il s'agit de jouer avec la vie et les souffrances de ses victimes. Des points de vue similaires se retrouvent également dans les chansons rromanies de Roumanie. Une partie de cet article est consacrée aux noms propres turcs utilisés par les Rroms et une autre aux expressions et locutions turques présentes dans les chansons. L'étude englobe l'édition bilingue de onze chansons rromanies de Bulgarie et une de Roumanie, dans lesquelles est dépeint le personnage du Turc.

Due to the late access of Rromani to written literature (after WWII in the Balkan), only oral accounts can provide illustrative material for research about such and such character in Rromani cosmovision. This is true among others for the Rroms' views on the Turks' character. After explaining the various names given to the Turks in Rromani folk songs, the presentation attempts to define the main features of this character, as mirrored in these songs: mercilessness, fierceness, even sadism in playing with their victim's life and sufferings. Similar views are encountered in Rromani folk songs from Rumania as well. A passage of the article is devoted to Turkish proper names used among Rroms and another to Turkish phrases and expressions embodied in the songs. The study encloses the bilingual edition of 11 Rromani songs from Bulgaria and one from Rumania, in which the character of the Turk is depicted.

\section{INDEX}

Index géographique : Balkans, Bulgarie, Roumanie

motsclestr Bulgaristan, Romanya, Balkanlar, Osmanlı İmparatorluğu

motsclesmk БАЛКАНОТ, БУГАРИЈА, ОТОМАНСКАТА ИМПЕРИЈА, РОМАНИЈА

Mots-clés : Boša, chants populaires, littérature rromanie, Romanov Manus, culture populaire, Rroms, Ottomans dans la littérature

Keywords : folk songs, Rrom, turk, Balkans, Bulgaria, Rumania, Ottoman empire, Orale

literature, Literature

Thèmes : Littérature, Littérature orale

Index chronologique : Empire ottoman

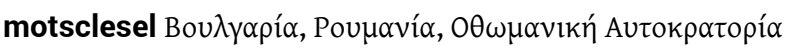

